\title{
NONLINEAR DYNAMICS IN AN EXTENDED NEIGHBOURHOOD OF THE TRANSLUNAR EQUILIBRIUM POINT
}

\author{
ÀNGEL JORBA AND JOSEP MASDEMONT \\ Departament de Matemàtica Aplicada I (ETSEIB) \\ Universitat Politècnica de Catalunya \\ Diagonal 64\%, 08028 Barcelona (Spain)
}

\section{Introduction}

We are interested in the motion of a small particle in some regions of the Earth-Moon system. As a first model, we will use the spatial Restricted Three Body Problem (RTBP, see [5] for definition and main properties). It is well known that, in synodic coordinates, this model has five equilibrium points. We will focus on the one that is behind the Moon, usually called the $L_{2}$ point. Our purpose is to describe the dynamics in an extended neighbourhood of that point. This information is very useful to keep an spacecraft there, because we can take advantage of the natural dynamics of the problem.

The main tools used are an effective computation of the central manifold of the point (to give a qualitative description of the dynamics) and a Lindstedt-Poincaré method (to compute invariant tori inside the central manifold). This methodology has already been used in similar problems (see [1]).

\section{Effective reduction to the central manifold}

This section is devoted to the computation of the central manifold associated to $L_{2}$. The procedure is quite similar to a normal form computation.

\subsection{THE HAMILTONIAN}

The reference system is the following: the origin is taken at $L_{2}$, the $x$ axis is directed opposite to the Moon, the $z$ axis is given by the vector of angular motion of Earth and Moon and the $y$ axis is selected in order to obtain a orthogonal positive-oriented system of reference. Then, the Hamiltonian takes the form

$$
H=\frac{1}{2}\left(p_{x}^{2}+p_{y}^{2}+p_{z}^{2}\right)+y p_{x}-x p_{y}-\frac{1-\mu}{r_{E}}-\frac{\mu}{r_{M}},
$$

where $r_{E}$ and $r_{M}$ are the distances from $(x, y, z)$ to Earth and Moon respectively. To compute the power expansion of $H$ around the origin, it is worth noting that

$$
\frac{1-\mu}{r_{E}}+\frac{\mu}{r_{M}}=\sum_{n \geq 2} c_{n} \rho^{n} P_{n}\left(\frac{x}{\rho}\right)
$$


where $\rho^{2}=x^{2}+y^{2}+z^{2}, P_{n}$ is the Legendre polynomial of degree $n$ and

$$
c_{n}=\frac{(-1)^{n}}{\gamma^{3}}\left[\mu+(1-\mu)\left(\frac{\gamma}{1+\gamma}\right)^{n+1}\right] \text {, }
$$

being $\gamma$ the distance between $L_{2}$ and Moon divided by the Earth-Moon distance. A good way of implementing this expansion on a computer is to take advantage of the recurrence of the Legendre polynomials (see, for instance, [2]).

\subsection{LINEAR BEHAVIOUR AND EXPANSIONS}

The linearization of the Hamiltonian around $L_{2}$ shows that the local behaviour near this point is of the type saddle $\times$ centre $\times$ centre. So, using a real, linear and symplectic change of variables the Hamiltonian takes the form

$$
H=\lambda x_{1} y_{1}+\frac{\omega_{1}}{2}\left(x_{2}^{2}+y_{2}^{2}\right)+\frac{\omega_{2}}{2}\left(x_{3}^{2}+y_{3}^{2}\right)+\cdots,
$$

where $\lambda, \omega_{1}$ and $\omega_{2}$ are real numbers. For the following computations it is very convenient to "diagonalize" the second order terms. This is done by using the complex change $x_{i}=$ $\left(q_{i}+\sqrt{-1} p_{i}\right) / \sqrt{2}, y_{i}=\left(\sqrt{-1} q_{i}+p_{i}\right) / \sqrt{2}, i=2,3$. So, the Hamiltonian looks like

$$
H=\lambda q_{1} p_{1}+\sqrt{-1} \omega_{1} q_{2} p_{2}+\sqrt{-1} \omega_{2} q_{3} p_{3}+\sum_{m \geq 3} \sum_{|k|=m} h_{k} q_{1}^{k_{1}} p_{1}^{k_{2}} q_{2}^{k_{3}} p_{2}^{k_{4}} q_{3}^{k_{5}} p_{3}^{k_{6}} .
$$

In fact, a good way of performing these changes of variables on the Hamiltonian is to write them as a unique linear transformation and to apply the recurrence mentioned above to this change. This produces the expansion of the Hamiltonian in the final variables.

\subsection{COMPUTATION OF THE CENTRAL MANIFOLD}

The next step is to perform canonical transformations to (2) to obtain

$$
H=\lambda q_{1} p_{1}+\sqrt{-1} \omega_{1} q_{2} p_{2}+\sqrt{-1} \omega_{2} q_{3} p_{3}+\sum_{m \geq 3} \sum_{\substack{|k|=m \\ k_{1}=k_{2}}} h_{k} q_{1}^{k_{1}} p_{1}^{k_{2}} q_{2}^{k_{3}} p_{2}^{k_{4}} q_{3}^{k_{5}} p_{3}^{k_{6}} .
$$

The changes are done using Poisson brackets (see, for similar examples, [3], [4] or [2]). The purpose is to kill all the monomials with $k_{1} \neq k_{2}$. Of course, this is done up to a finite order $N$. The divisors obtained in the generating function of the change of variables are

$$
\lambda\left(k_{2}-k_{1}\right)+\sqrt{-1} \omega_{1}\left(k_{4}-k_{3}\right)+\sqrt{-1} \omega_{2}\left(k_{6}-k_{5}\right) .
$$

As we only want to eliminate monomials with $k_{1} \neq k_{2}$, the corresponding divisors can be bounded from below. This shows the absence of small denominators in the process. So, although this procedure is not convergent, it produces very good approximations to the dynamics. The last step is to call $I=q_{1} p_{1}$, and the final Hamiltonian looks like

$$
H=H_{N}\left(I, q_{2}, p_{2}, q_{3}, p_{3}\right)+R_{N+1}(q, p) .
$$

Now, if we skip $R_{N+1}$ (it is very small near $L_{2}$ ), we see that $I$ is a first integral, and setting $I=0$ we skip the hyperbolic part and we reduce the Hamiltonian to its central manifold. Finally, the resulting Hamiltonian is realified. 


\section{Dynamics inside the central manifold}

The phase space in the central manifold is four dimensional. To describe the dynamics, we fix the Poincaré section $q_{3}=0$ (this will correspond, at first order, to $z=0$ in the initial coordinates) and we use, as a parameter, the energy level $h$ of the Hamiltonian. This implies that it is enough to plot these 2-D sections for several levels of $h$ to obtain a qualitative description of the phase space.

\section{Computation of invariant tori near $L_{2}$}

Here is better to work with the equations of motion instead of the Hamiltonian. They can be written in the from

$$
\begin{aligned}
\ddot{x}-2 \dot{y}-\left(1+2 c_{2}\right) x & =\frac{\partial}{\partial x} \sum_{n \geq 3} c_{n} \rho^{n} P_{n}\left(\frac{x}{\rho}\right), \\
\ddot{y}+2 \dot{x}+\left(c_{2}-1\right) y & =\frac{\partial}{\partial y} \sum_{n \geq 3} c_{n} \rho^{n} P_{n}\left(\frac{x}{\rho}\right), \\
\ddot{z}+c_{2} z & =\frac{\partial}{\partial z} \sum_{n \geq 3} c_{n} \rho^{n} P_{n}\left(\frac{x}{\rho}\right),
\end{aligned}
$$

where the reference system is the one introduced in section 2.1 and $c_{n}$ has been defined in (1). Note that, in these equations, the left-hand side contains the linear part and the right-hand side the nonlinear part. For the computation of invariant tori, we will use a LindstedtPoincaré method (see [1]). This is a recurrent procedure that produces approximations to the solutions starting from the solutions of the linear part.

\subsection{THE 2-D INVARIANT TORI}

We look for formal expansions, in powers of the amplitudes $\alpha$ and $\beta$, of the type

$$
\begin{aligned}
x & =\sum_{i, j=1}^{\infty}\left(\sum_{k \leq|i|, m \leq|j|} a_{i j k m} \cos \left(k \theta_{1}+m \theta_{2}\right)\right) \alpha^{i} \beta^{j}, \\
y & =\sum_{i, j=1}^{\infty}\left(\sum_{k \leq|i|, m \leq|j|} b_{i j k m} \sin \left(k \theta_{1}+m \theta_{2}\right)\right) \alpha^{i} \beta^{j}, \\
z & =\sum_{i, j=1}^{\infty}\left(\sum_{k \leq|i|, m \leq|j|} c_{i j k m} \cos \left(k \theta_{1}+m \theta_{2}\right)\right) \alpha^{i} \beta^{j},
\end{aligned}
$$

where $\theta_{1}=\omega t+\Phi_{1}$ and $\theta_{2}=\nu t+\Phi_{2}$. The frequencies $\omega$ and $\nu$ are expanded as $\omega=$ $\sum_{i, j=0}^{\infty} \omega_{i j} \alpha^{i} \beta^{j}$ and $\nu=\sum_{i, j=0}^{\infty} \nu_{i j} \alpha^{i} \beta^{j}$. All the coefficients are computed recurrently up to a finite order $i+j$.

\subsection{HALO ORBITS}

Now we look for (periodic) Halo orbits. In this case we only have one frequency and one amplitude. Nevertheless, we will keep the two amplitudes $\alpha$ and $\beta$ adding a constrain 
between them. So, the expansions have the form

$$
\begin{aligned}
& x=\sum_{i, j=1}^{\infty}\left(\sum_{k \leq i+j} a_{i j k} \cos (k \omega t)\right) \alpha^{i} \beta^{j}, \\
& y=\sum_{i, j=1}^{\infty}\left(\sum_{k \leq i+j} b_{i j k} \sin (k \omega t)\right) \alpha^{i} \beta^{j}, \\
& z=\sum_{i, j=1}^{\infty}\left(\sum_{k \leq i+j} c_{i j k} \cos (k \omega t)\right) \alpha^{i} \beta^{j},
\end{aligned}
$$

where $\omega=\sum_{i, j=0}^{\infty} \omega_{i j} \alpha^{i} \beta^{j}$ and the relation between both amplitudes can be written in the form $\Delta=\sum_{i, j=0}^{\infty} \Delta_{i j} \alpha^{i} \beta^{j}=0$.

We want to stress that Halo orbits are not present in the linearized system and they are only found when nonlinear terms are considered, since they are produced by a 1 to 1 resonance between the two frequencies of the central part.

\section{Results}

All the algorithms have been implemented by the authors in $\mathrm{C}$ and FORTRAN, taking advantage of the symmetries and particularities of the problem. The use of commercial algebraic manipulators does not allow to reach high orders in the expansions to get accurate results (the central manifold has been computed up to order 32, the Lindstedt-Poincaré has been applied up to order 35 for the invariant tori and 45 for the Halo orbits).

We have summarized the results in figure 1. The first plot is one of the Poincare sections $(h=1)$ of the central manifold. The elliptic point in the centre corresponds to a vertical Lyapunov orbit, the lateral elliptic points correspond to Halo orbits and the boundary of the section is a planar Lyapunov orbit.

The second plot corresponds to one of the invariant tori in the central manifold. It has been plotted as seen from the Earth, jointly with the Moon disk. Note the "cut" between the initial and the final part of the trajectory, just over the Moon disk. This point has the same position in both cases but with different velocities. This implies that, if we want to avoid the lunar disk, we can restart the orbit with only a manoeuvre. The time between two of these manoeuvres is about two weeks.

\section{Applications to spacecraft dynamics}

The most interesting trajectories are the ones that, as seen from the Earth, avoid the Moon disk. They can be useful, for instance, to keep constant communication with a mission to the dark side of the Moon. Halo orbits are suitable for that purpose. The main drawback is that the orbit is very wide in the horizontal direction, so the spacecraft must carry an orientable antenna, always pointing to the Earth. This makes the mission more expensive.

Another possibility is to use an invariant tori in the central manifold of $L_{2}$. Note that the orbit can be selected more "squared", so the antenna does not need to be orientable. On the other hand, and from time to time, the spacecraft will be eclipsed by the Moon. This can be avoided by doing a manoeuvre every two weeks.

Full details of these computations, as well as the stability properties of the orbits and the corresponding control strategies will appear in a future paper. 

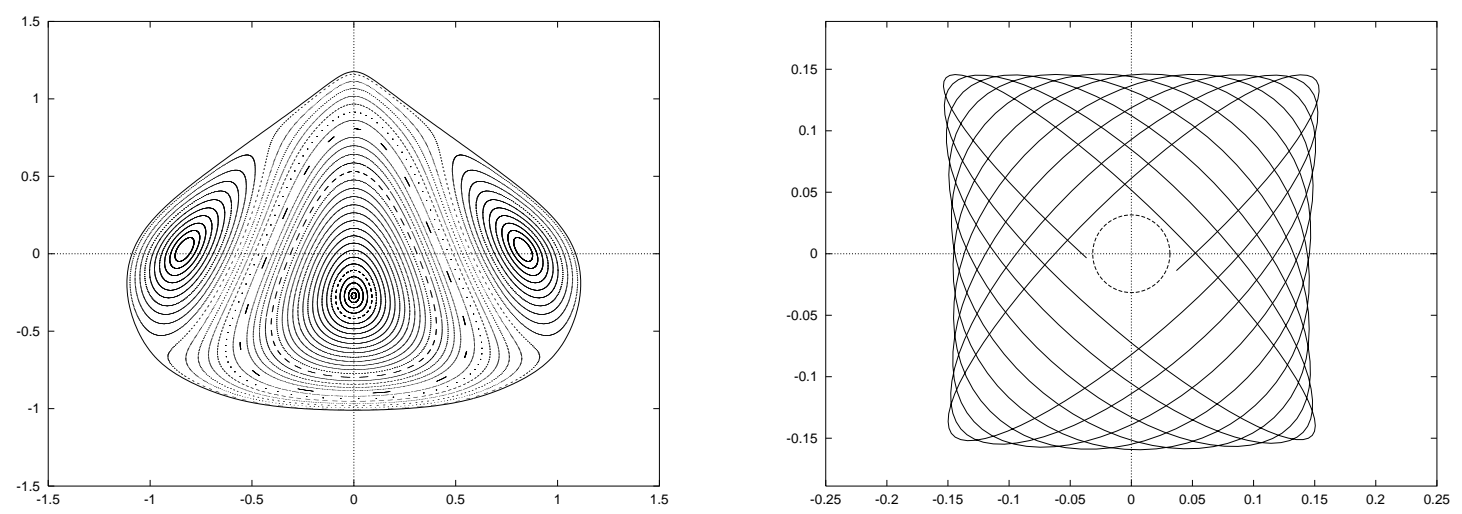

Figure 1. Left: Poincaré section of the central manifold. Right: Quasiperiodic trajectory in the central manifold, as seen from the Earth (in the centre we have drawn the Moon disk).

\section{Extensions}

The next step is to take into account the main perturbations of the problem, namely the noncircular motion of the Moon and the effect of the Sun. This will allow to give precise details about the dynamics of the real problem. This work is still in progress.

\section{Acknowledgements}

A. Jorba has been partially supported by the grants DGICYT PB94-0215, CIRIT GRQ931135, the European Network ERBCHRXCT 940460 and the UPC fund PR9409. J. Masdemont has been supported by the grant DGICYT PB94-0215 and the UPC fund PR9409.

\section{References}

1. Gómez, G., Jorba, A., Masdemont, J. and Simó, C. (1991) Study refinement of semianalytical halo orbit theory, ESOC Contract 8625/89/D/MD(SC), Final Report.

2. Jorba, A. and Simó, C. (1994) Effective stability for periodically perturbed Hamiltonian systems, in Hamiltonian Mechanics, Integrability and Chaotic Behavior, J. Seimenis (Ed.), Plenum Press, New York, pp. 245-252.

3. Giorgilli, A., Delshams, A., Fontich, E., Galgani, L. and Simó, C. (1989) Effective stability for a Hamiltonian system near an elliptic equilibrium point, with an application to the Restricted Three Body Problem, J. of Diff. Equations, 77:1, pp. 167-198.

4. Simó, C. (1989) Estabilitat de sistemes Hamiltonians, Mem. de la Real Acad. de Ciencias y Artes de Barcelona, Vol. XLVIII, no. 7, pp. 303-348.

5. Szebehely, V. (1967) Theory of orbits, Academic Press. 